\title{
Xylo-oligosaccharides alone or in synbiotic combination with Bifidobacterium animalis subsp. lactis induce bifidogenesis and modulate markers of immune function in healthy adults: a double-blind, placebo-controlled, randomised, factorial cross-over study
}

\author{
Caroline E. Childs ${ }^{1,2_{*}}$, Henna Röytiö ${ }^{3,4}$, Esa Alhoniemi ${ }^{5}$, Agnes A. Fekete ${ }^{1}$, Sofia D. Forssten ${ }^{3}$, \\ Natasa Hudjec ${ }^{1}$, Ying Ni Lim ${ }^{1}$, Cara J. Steger ${ }^{1}$, Parveen Yaqoob $^{1}$, Kieran M. Tuohy ${ }^{1,6}$, Robert A. Rastall ${ }^{1}$, \\ Arthur C. Ouwehand ${ }^{3}$ and Glenn R. Gibson ${ }^{1}$ \\ ${ }^{1}$ Department of Food and Nutritional Sciences, The University of Reading, Reading RG6 6AP, UK \\ ${ }^{2}$ Human Development and Health Academic Unit, Faculty of Medicine, University of Southampton, IDS Building, \\ MP887 Southampton General Hospital, Tremona Road, Southampton SO16 6YD, UK \\ ${ }^{3}$ Active Nutrition, DuPont Nutrition and Health, Kantvik. FI-02460, Finland \\ ${ }^{4}$ University of Turku, Functional Foods Forum, Turku FI-20520, Finland \\ ${ }^{5}$ Pharmatest Services Limited, Turku FI-20520, Finland \\ ${ }^{6}$ Department of Food Quality and Nutrition, Research and Innovation Centre, Fondazione Edmund Mach, \\ via E. Mach, 1, San Michele all'Adige, Trento 38010, Italy
}

(Submitted 12 April 2013 - Final revision received 15 November 2013 - Accepted 29 November 2013 - First published online 24 March 2014)

\section{Abstract}

Prebiotics, probiotics and synbiotics are dietary ingredients with the potential to influence health and mucosal and systemic immune function by altering the composition of the gut microbiota. In the present study, a candidate prebiotic (xylo-oligosaccharide, XOS, $8 \mathrm{~g} / \mathrm{d}$ ), probiotic (Bifidobacterium animalis subsp. lactis $\mathrm{Bi}-07,10^{9}$ colony-forming units $\left.(\mathrm{CFU}) / \mathrm{d}\right)$ or synbiotic $\left(8 \mathrm{~g} \mathrm{XOS}+10^{9} \mathrm{CFU} \mathrm{Bi}-07 / \mathrm{d}\right)$ was given to healthy adults (25-65 years) for $21 \mathrm{~d}$. The aim was to identify the effect of the supplements on bowel habits, self-reported mood, composition of the gut microbiota, blood lipid concentrations and immune function. XOS supplementation increased mean bowel movements per $\mathrm{d}(P=0.009)$, but did not alter the symptoms of bloating, abdominal pain or flatulence or the incidence of any reported adverse events compared with maltodextrin supplementation. XOS supplementation significantly increased participant-reported vitality $(P=0.003)$ and happiness $(P=0.034)$. Lowest reported use of analgesics was observed during the XOS + Bi- 07 supplementation period $(P=0.004)$. XOS supplementation significantly increased faecal bifidobacterial counts $(P=0.008)$ and fasting plasma HDL concentrations $(P=0 \cdot 005)$. Bi-07 supplementation significantly increased faecal $B$. lactis content $(P=0 \cdot 007)$, lowered lipopolysaccharide-stimulated IL-4 secretion in whole-blood cultures $(P=0.035)$ and salivary IgA content $(P=0.040)$ and increased IL-6 secretion $(P=0.009)$. XOS supplementation resulted in lower expression of CD16/56 on natural killer T cells $(P=0 \cdot 027)$ and lower IL-10 secretion $(P=0 \cdot 049)$, while XOS and Bi-07 supplementation reduced the expression of $\mathrm{CD} 19$ on $\mathrm{B}$ cells (XOS $\times \mathrm{Bi}-07, P=0.009$ ). The present study demonstrates that XOS induce bifidogenesis, improve aspects of the plasma lipid profile and modulate the markers of immune function in healthy adults. The provision of XOS $+\mathrm{Bi}-07$ as a synbiotic may confer further benefits due to the discrete effects of Bi-07 on the gut microbiota and markers of immune function.

Key words: Pre-, pro- and synbiotics: Gut microbiota: Immune function

A prebiotic is 'a selectively fermented ingredient that results in specific changes in the composition and/or activity of the gastrointestinal microbiota, thus conferring benefit(s) upon host health $^{\text {,1) }}$. Xylo-oligosaccharides (XOS) are relatively stable under acidic conditions, which may endow protection from digestion when passing through the stomach ${ }^{(2)}$. The degradation of xylobiose (XOS degree of polymerisation $=2$ ) in the intestine has been studied in vitro and it has been found that XOS may be non-digestible and would reach the colon intact after oral intake ${ }^{(3)}$

The preferential fermentation of XOS by bifidobacteria, including $B$. lactis, has been demonstrated in vitro through pure culture studies ${ }^{(4-9)}$ and faecal batch and semi-continuous

Abbreviations: Bi-07, Bifidobacterium animalis subsp. lactis; FITC, fluorescein isothiocyanate; MDX, maltodextrin; NKT, natural killer T; PE, phycoerythrin; Th, T helper; XOS, xylo-oligosaccharides.

* Corresponding author: Dr C. E. Childs, fax + 442381 204221, email c.e.childs@soton.ac.uk 
mixed-culture fermentations ${ }^{(10-12)}$. Animal studies have demonstrated that XOS stimulate the growth of caecal and faecal bifidobacteria at higher levels compared with the prebiotic fructo-oligosaccharide ${ }^{(13-15)}$. In human studies, $2-5 \mathrm{~g}$ of XOS/ $\mathrm{d}$ have been found to result in significant increases in the population of faecal bifidobacteria and faecal concentrations of SCFA and concomitant decreases in $\mathrm{pH}$, proteolytic metabolite levels and enzyme activity ${ }^{(16-18)}$ and to have an ameliorating effect on constipation in women ${ }^{(19,20)}$. Therefore, evidence for the prebiotic capacity and health benefits of XOS is promising, but further placebo-controlled studies are required, in particular, to evaluate a wide range of health parameters ${ }^{(21,22)}$.

Prebiotics may influence immune function by altering the profile of pathogen-associated molecular patterns presented to the gut-associated lymphoid tissue or via indirect effects of microbial metabolic products, such as SCFA ${ }^{(23)}$. Dietary XOS have been shown to significantly increase resistance to Listeria monocytogenes in a guinea pig model ${ }^{(24)}$, with in vitro studies confirming that XOS decrease the adherence of this pathogen to intestinal epithelial cells ${ }^{(25)}$. Using $5 \mathrm{~g} / \mathrm{d}$ XOS, a human study has identified a bifidogenic effect, but no significant effects upon the markers of immune function measured (lipopolysaccharide-induced cytokine production and faecal secretory IgA content) have been observed ${ }^{(18)}$.

The positive effects of probiotics on immune function have been observed in animal studies and trials in children, adults and the elderly, but influences are species and strain specific and variable ${ }^{(26)}$. Placebo-controlled studies using Bifidobacterium animalis subsp. lactis (Bi-07) have identified reduced incidence of bloating in adults with functional bowel disorders $^{(27)}$ and cold and influenza-like symptoms in children ${ }^{(28)}$.

The provision of synbiotics (prebiotics combined with a probiotic) may increase probiotic survival after consumption and ensure persistence of the probiotic strain within the gut microbiota $^{(29)}$. However, studies using synbiotic preparations often have the limitation that it remains unclear whether any effect observed is a result of the prebiotic or probiotic or a synergistic effect of the combined supplement.

The present double-blind, placebo-controlled cross-over study investigated the effect of XOS (provided as a prebiotic and/or in combination with Bi-07) in healthy adults. It is our hypothesis that the provision of XOS in combination with Bi-07 will be advantageous over individual provision of these supplements due to a synergistic effect arising from

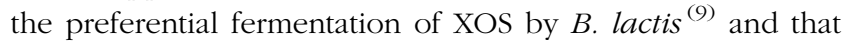
beneficial effects will include changes to the composition of the gut microbiota and immune function. The primary outcome measure was the effect of supplements on faecal bifidobacterial content. The secondary outcome measures were the effects of supplements on the gut microbiota and faecal SCFA concentrations, bowel habits, self-reported mood, plasma lipids and immune function markers. The assessment of immune function is complex, requiring multiple markers to be monitored to fully assess the potential impact of a nutritional intervention. In the present study, markers selected for analysis included those reflecting systemic and mucosal immunity, innate and acquired immunity, cellular activity and concentrations of soluble mediators.

\section{Experimental methods}

\section{Subjects}

The study group size required was estimated using G*Power $3.0 .10^{(30)}$. On the basis of $5 \%$ significance, $95 \%$ power and 0.5 correlation between the groups for dependent means, a sample size of 7 would be required to detect an effect of XOS supplementation on the primary outcome (increased levels of Bifidobacterium population in faeces $\left.{ }^{(17)}\right)$. Data from human studies that assessed the effects of probiotics $^{(31,32)}$, prebiotics $^{(33)}$ or synbiotics ${ }^{(34)}$ on immune function were used to determine the sample size required to detect significant effects on immune function including markers of phagocytosis, lymphocyte subsets and cytokine production. The effect sizes observed in these available human studies ranged from 0.3 to $1 \cdot 7$, with a variance of $0 \cdot 2$. On the basis of $5 \%$ significance, $95 \%$ power and 0.5 correlation between the groups for dependent means, the median sample size required for nine assessed immune outcomes was 27 , with six/nine outcomes requiring $n<35$. Therefore, we aimed to recruit forty volunteers to complete the study and recruited forty-four volunteers to allow for participant dropout to assess significant effects on both primary and secondary outcomes. Therefore, the minimum detectable effect size would be 0.58 (on the basis of $5 \%$ significance and $95 \%$ power).

The present study was conducted according to guidelines laid down in the Declaration of Helsinki, and all procedures involving human subjects were approved by the Ethics and Research Committee of the University of Reading. Written informed consent was obtained from all subjects. Verbal consent was witnessed and formally recorded. In total, forty-four volunteers were recruited from the Reading area between September 2008 and January 2009, and the study was completed in June 2009. Inclusion criteria included the following: a signed consent form; age 25-65 years; BMI $20-30 \mathrm{~kg} / \mathrm{m}^{2}$; good general health, as determined by medical questionnaire. Exclusion criteria included the following: evidence of physical or mental disease requiring inpatient/outpatient treatment and/or use of prescription medication; planned major surgery; history of drug or alcohol abuse; severe allergies or a history of severe abnormal drug reaction; participation in an experimental drug trial 4 weeks before the study; participation in prebiotic or laxative trials in the previous 3 months; use of antibiotics in the previous 6 months; chronic constipation, diarrhoea or other chronic gastrointestinal complaints; use of other prebiotics or probiotics in the previous 4 weeks, drugs active on gastrointestinal motility, or a laxative of any class for 4 weeks before study; use of prescribed medication; regular use of aspirin or other anti-inflammatory drugs.

\section{Study design}

Dietary supplements were given to the volunteers in the present double-blind, placebo-controlled, randomised, factorial cross-over study (Clinicaltrials.gov identifier: NCT01545219). The supplements were provided for $21 \mathrm{~d}$, with a $28 \mathrm{~d}$ washout period. The supplements were XOS $(8 \mathrm{~g} / \mathrm{d}$; Shandong Long-live Biotech), B. lactis (Bi-07 ATCC SD5220, 109 
colony-forming units/d; Danisco) and the control maltodextrin (MDX; Syral). MDX was selected for use as the "placebo control' as it is fully absorbed as glucose within the small intestine and therefore will not influence the gut microbiota. The volunteers were given two sachets of daily supplements in powder form and advised to dissolve the contents together in water, milk or fruit juice. In the present double-blind study, all supplements were identically packaged and identified by an alphabetic code. The volunteers were asked to refrain from consuming any other probiotic or prebiotic product during the study period and given advice on common dietary products containing supplemental probiotics or prebiotics. The volunteers were given additional sachets at the beginning of each supplementation period and were asked to return unused sachets as a marker of compliance. The volunteers were randomised by sex, age and BMI to their starting point in the supplement sequence $(\mathrm{MDX}+\mathrm{MDX}, \mathrm{XOS}+\mathrm{MDX}, \mathrm{Bi}-07+\mathrm{MDX}$ and $\mathrm{XOS}+\mathrm{Bi}-07)$ by covariate adaptive randomisation ${ }^{(35)}$. Staff responsible for enrolling participants, assigning participants to the supplement sequence and assessing outcomes remained blinded to treatment identity until data analysis was completed. Before the start of the study, the volunteers completed a $4 \mathrm{~d}$ food diary, which was analysed using DietPlan6.60b (Forestfield Software Limited). The volunteers attended study appointments before and after each supplementation or washout period. During study appointments, anthropometric measurements were recorded (weight, blood pressure and waist circumference) and a fasting blood sample and samples of saliva and faeces were collected from the volunteers.

\section{Faecal sample processing}

Freshly voided faecal samples were collected in a sterile plastic pot at the start and end of each treatment and washout period. The samples collected for faecal dry weight and IgA assays were stored at $-20^{\circ} \mathrm{C}$, and those collected for quantitative PCR analyses and enumeration of total bacteria by flow cytometry were stored at $-80^{\circ} \mathrm{C}$. The remaining faecal samples were diluted 1 in $10(\mathrm{w} / \mathrm{w})$ in PBS (0.1 M; pH 7.0) and homogenised in Stomacher 400 (Seward) for $2 \mathrm{~min}$ at normal speed (460 paddle beats/min). A $15 \mathrm{ml}$ sample of faecal slurry was vortexed with $2 \mathrm{~g}$ of $3 \mathrm{~mm}$-diameter glass beads (VWR) and then centrifuged to remove particulate matter ( $1500 \mathrm{~g}, 2 \mathrm{~min}$ ). The supernatant was collected for the determination of SCFA concentrations and assessment of genus-level changes in the gut microbiota by fluorescence in situ hybridisation with $16 \mathrm{~S}$ rRNA-targeted oligonucleotide probes.

\section{Fluorescence in situ hybridisation}

Faecal slurry supernatants were fixed in paraformaldehyde ( $1: 4, \mathrm{v} / \mathrm{v}$ in $4 \%$ paraformaldehyde in $0.1 \mathrm{M}-\mathrm{PBS}, \mathrm{pH} 7 \cdot 2$ ) for $4 \mathrm{~h}$ at $4^{\circ} \mathrm{C}$, centrifuged $(13000 \mathrm{~g} 5 \mathrm{~min})$, washed twice with $0 \cdot 1 \mathrm{M}$-PBS, resuspended in 1:1 PBS-ethanol and stored at $-20^{\circ} \mathrm{C}$. Oligonucleotide probes used were Cy-3 labelled and synthesised by Sigma-Aldrich. Probes used were Bif164, Bac303, Chis150, Lab158 and ATO291 specific for Bifidobacterium spp., Bacteroides/Prevotella group, Clostridium clusters I and II (including C. perfringens and C. histolyticum), Lactobacillus/Enterococcus subgroup and Atopobium, respectively. Samples were hybridised as described previously ${ }^{(36)}$. Data are expressed as $\log _{10}$ counts/g dry-weight faeces.

\section{Quantification of Bifidobacterium lactis}

DNA was extracted from the faecal samples using the QIAamp DNA Stool Mini Kit (Qiagen) following the manufacturer's instructions. Quantitative PCR was carried out for the quantification of $B$. lactis using the FAST SYBR Green methodology (Applied Biosystems) in a total volume of $25 \mu$ l containing $1 \mathrm{ng}$ of template DNA and $250 \mathrm{~nm}$ of the forward primer Blact_ $1^{(12)}$ and the reverse primer Bflact $5^{(37)}$. The amplification and detection of DNA were carried out with an ABI 7500 sequencing detection system (Applied Biosystems). To obtain a standard curve, a 10-fold dilution series ranging from $10 \mathrm{pg}$ to $10 \mathrm{ng}$ of DNA from the bacterial standard culture of B. lactis (ATCC SD5220) was included in the PCR assays. For the determination of DNA content, triplicate samples were used, and the mean quantity/g dry weight was calculated.

\section{Quantification of total bacteria by flow cytometry}

Total bacteria in faecal samples were quantified using a flow cytometric FACSCalibur system (BD Biosciences) as described previously ${ }^{(38)}$. Frozen faecal samples were thawed, and bacteria were recovered from the faecal samples by diluting and washing the samples 1:30 with washing buffer $(50 \mathrm{~mm}$ sodium phosphate buffer, $\mathrm{pH} 8$ ) on a reciprocating horizontal platform shaker at $200 \mathrm{rpm}$ for $10 \mathrm{~min}$ and then centrifuging at $30000 \mathbf{g}$ for $30 \mathrm{~min}$ at room temperature. The supernatant was discarded, and the pellet was washed three more times as described above. A subsample from the suspension was withdrawn before the last centrifugation, fixed with $4 \%$ formaldehyde and stained with a fluorescent, nucleic acid-binding dye, SYTO 24 (Molecular Probes). The total number of bacterial cells was determined by comparing the cellular events with the bead events in BD Trucount ${ }^{\mathrm{TM}}$ tubes (BD Biosciences).

\section{Determination of SCFA concentrations}

Faecal slurry supernatants were used to determine the faecal concentrations of SCFA including acetic acid, propionic acid, $i$-butyric acid, $n$-butyric acid, $i$-valeric acid, $n$-valeric acid, $n$-caproic acid and $\mathrm{D} / \mathrm{L}$-lactic acid by $\mathrm{GC}^{(39)}$. Thawed faecal slurry supernatants were centrifuged at $13000 \boldsymbol{g}$ for $10 \mathrm{~min}$. To $0.5 \mathrm{ml}$ aliquots of centrifuged faecal slurry supernatants, $25 \mu \mathrm{l}$ of an internal standard ( $100 \mathrm{~mm}$ of 2-ethyl butyric acid), $25 \mu \mathrm{l}$ of concentrated $\mathrm{HCl}$ and $1 \mathrm{ml}$ of diethyl ether were added and centrifuged at $3000 \mathrm{~g}$ for $10 \mathrm{~min}$. Later, $400 \mu \mathrm{l}$ of the resulting upper ether layer were combined with $50 \mu \mathrm{l}$ of $N$-tert-butyldimethylsilyl- $N$-methyltrifluoroacetamide, heated at $80^{\circ} \mathrm{C}$ for $20 \mathrm{~min}$ in a water-bath and then left at room temperature for $48 \mathrm{~h}$ to allow derivatisation.

The samples were run on a 5890 series II Hewlett Packard GC system (HP) using a dimethylpolysiloxane column $(10 \mathrm{~m} \times 0 \cdot 18 \mathrm{~mm} \times 0 \cdot 20 \mu \mathrm{m}$ film thickness; Thomas Restek) 
and detected with a flame ionisation detector. Helium was used as the carrier gas, and samples $(1 \mu \mathrm{l})$ were injected at a run time of $15 \mathrm{~min}$. The detector was set to $275^{\circ} \mathrm{C}$, and the temperature of the oven was held at $60^{\circ} \mathrm{C}$ for $3 \mathrm{~min}$, then increased $10^{\circ} \mathrm{C} / \mathrm{min}$ to $150^{\circ} \mathrm{C}$ and held for $3 \mathrm{~min}$. Chemstation REV.A.10.01 software (Agilent Technologies) was used to integrate peaks. The concentrations of SCFA were determined by comparing their peak areas with external standards, relative to the internal standard. The concentrations of fatty acids are expressed as $\mu \mathrm{mol} / \mathrm{g}$ of wet faeces.

\section{Volunteer bowel habit and mood questionnaires}

During the supplementation and washout periods, the volunteers were given a daily diary to record their bowel habits and mood, use of medication and adverse events. The volunteers recorded the number of bowel movements per $\mathrm{d}$, Bristol stool scale score and any symptoms of bloating or abdominal discomfort (rated as none, mild, moderate or severe) ${ }^{(40)}$. The volunteers were asked to rate their flatulence on a five-point scale (with a score of 0 for 'normal', +1 or +2 for more than usual, and -1 or -2 for less than usual). Self-reported mood was also assessed using a five-point scale, with vitality, stress, happiness and alertness being recorded. Mean reported scores and the percentage of days during which 'more than usual' or 'less than usual' of any symptom were reported during each supplementation period were used for statistical analyses.

\section{Quantification of plasma lipid concentrations}

Plasma total cholesterol, HDL-cholesterol, LDL-cholesterol, TAG and NEFA concentrations were quantified with an automated clinical chemistry analyser (Instrumentation Laboratory Limited) using enzyme-based colorimetric kits supplied by Instrumentation Laboratory and Alpha Laboratories in accordance with the manufacturer's instructions.

\section{Determination of faecal and salivary IgA content}

Faecal and salivary IgA content was determined by ELISA (Immundiagnostik) in accordance with the manufacturer's instructions.

\section{Measurement of phagocytosis and oxidative burst}

Phagocytosis and oxidative burst by monocytes and granulocytes in fresh blood samples was measured using PHAGOTEST $^{\circledR}$ and BURSTTEST ${ }^{\circledR}$ (Orpegen Pharma) in accordance with the manufacturer's instructions.

\section{Determination of immune cell counts and phenotypes}

Total leucocyte counts were determined using the Beckman Coulter Z1 automated cell counter and ZAP-OGLOBIN II Lytic reagent (Beckman Coulter). Immune cell phenotyping was carried out on fresh blood samples using stains for $\mathrm{CD} 3$ fluorescein isothiocyanate (FITC)/CD4 phycoerythrin (PE), CD3 FITC/CD8
PE, CD3 FITC/CD16 PE + CD56 PE and CD3 FITC/CD19 PE obtained from BD Biosciences in accordance with the manufacturer's instructions to identify $\mathrm{T}$, T-helper (Th), cytotoxic T, natural killer, natural killer T (NKT) and B cells. Erythrocytes were lysed using PharmLyse (BD Biosciences), and samples were washed twice with a buffer solution (PBS, 1\% bovine serum albumin and $0 \cdot 1 \%$ sodium azide) and resuspended in a fixing solution ( $2 \%$ paraformaldehyde in PBS) before analysis. A total of 10000 events were collected on a FACSCalibur flow cytometer (BD Biosciences), and data were analysed using Flowjo 7.6.5 (Tree Star, Inc.).

\section{Measurement of lipopolysaccharide-stimulated cytokine production in whole-blood cultures}

For 24 h, 1:10 diluted whole-blood samples were incubated in the presence of $1 \mu \mathrm{g} / \mathrm{ml}$ lipopolysaccharide (Sigma-Aldrich). Culture supernatants were assessed for cytokine production using a Th1/Th 2 cytokine array (Bender MedSystems) comprising interferon- $\gamma$, IL-1 $\beta$, IL-2, IL-4, IL-5, IL-6, IL-8, IL-10, IL-12p70, TNF- $\alpha$ and TNF- $\beta$.

\section{Measurement of Concanavalin A-stimulated expression of CD69 on immune cells}

For 24 h, 1:10 diluted whole-blood samples were incubated in the presence of $50 \mu \mathrm{g} / \mathrm{ml}$ Concanavalin A (Sigma-Aldrich). CD69 (PE) expression on CD3-, CD4- and CD8 (FITC)-positive cells was determined using stains obtained from BD Biosciences in accordance with the manufacturer's instructions. Erythrocytes were lysed using PharmLyse (BD Biosciences), and samples were washed twice with a buffer solution (PBS, $1 \%$ bovine serum albumin and $0 \cdot 1 \%$ sodium azide) and resuspended in a fixing solution (2\% paraformaldehyde in PBS) before analysis. A total of 10000 events were collected on a FACSCalibur flow cytometer (BD Biosciences), and data were analysed using Flowjo 7.6.5.

\section{Statistical analyses}

For continuous measurements, change observed with the use of supplement $(\Delta)$ was considered (i.e. post-supplement value - pre-supplement value). Based on the residual analysis, some variables were $\log _{10}$-transformed before analysis. Data were analysed using linear mixed-effects models $(2 \times 2$ factorial approach) in a repeated-measures manner having a random effect for the subject accounting for repeated measures, continuous covariates for BMI, age, and fibre intake, fixed-effects terms for the presence/absence of the prebiotic and probiotic and their interaction (XOS, Bi-07, $\mathrm{XOS} \times \mathrm{Bi}-07$, a $2 \times 2$ factorial setting), and time point and starting point in the supplement sequence. Where significant effects of XOS, Bi-07 or XOS $\times$ Bi-07 were observed, pairwise post hoc comparisons with MDX were made using contrasts, adjusted using a single-step algorithm. Where significant carry-over effects were observed (fourteen/forty-eight variables for time point and one/forty-eight variables for starting point in the supplement sequence), only data from the first 
supplementation period were included in the final analysis. The questionnaire data were analysed using the Friedman test followed by Bonferroni corrected post hoc test based on mean rank differences between the supplements. Statistical significance was determined as $P<0.05$.

The analyses were conducted with R: A Language and Environment for Statistical Computing (version 2.14.2; $\mathrm{R}$ Development Core Team). The linear models were computed using the $\mathrm{R}$ package nlme: Linear and Nonlinear Mixed Effects Models (version 3.1-102; J Pinheiro, D Bates, S DebRoy, D Sarkar and R Development Core Team). The model contrasts were computed using the $\mathrm{R}$ package multcomp version $1.2 .12^{(41)}$.

\section{Results \\ Volunteer characteristics and compliance}

The characteristics of the volunteers recruited to the study are given in Table 1 . Of the forty-four volunteers recruited, forty-one completed the trial. The reasons for dropout were pregnancy (female: age 31 years), vasovagal reaction to blood sampling (male: age 57 years) and adverse reaction to the study product (female: age 64 years; probiotic). The adverse reaction to the probiotic occurred during the first supplementation period, with the volunteer reporting headache (days 1-7, 9, 11 and 12 of the supplementation period), abdominal pain, bloating and increased flatulence (days 6-12 of the supplementation period); the participant withdrew from the study on day 12 of the supplementation period.

There was good volunteer compliance with the study timetable, with $93 \%$ of the volunteers achieving supplement times of $21 \pm 1 \mathrm{~d}$ and $80 \%$ achieving washout periods of $28 \pm 1 \mathrm{~d}$. At the end of each supplementation period, the volunteers were asked to return any unused sachets to estimate compliance with supplement use. Compliance was estimated at $>90 \%$ among the thirty-five volunteers who returned unused sachets, with no significant differences being observed between the supplement groups. At the end of each supplementation period, the volunteers were asked whether they

Table 1. Baseline characteristics of the volunteers recruited to a double-blind, placebo-controlled, randomised cross-over study of a candidate prebiotic (xylo-oligosaccharide, XOS, $8 \mathrm{~g} / \mathrm{d}$ ), probiotic (Bifidobacterium animalis subsp. lactis, Bi-07, $10^{9}$ colony-forming units/d) or synbiotic (XOS $+\mathrm{Bi}-07$ ) (Mean values and standard deviations)

\begin{tabular}{|c|c|c|}
\hline & Mean & SD \\
\hline \multicolumn{3}{|l|}{$\operatorname{Sex}(n)$} \\
\hline Male & \multicolumn{2}{|c|}{22} \\
\hline Female & \multicolumn{2}{|c|}{22} \\
\hline Smokers $(n)$ & \multicolumn{2}{|c|}{$6 / 44$} \\
\hline Age (years) & 43 & 12 \\
\hline Height (m) & 1.7 & 0.1 \\
\hline Weight (kg) & 73 & 13 \\
\hline $\mathrm{BMI}\left(\mathrm{kg} / \mathrm{m}^{2}\right)$ & 25 & 3 \\
\hline Waist circumference $(\mathrm{cm})$ & 89 & 10 \\
\hline Systolic blood pressure (mmHg) & 126 & 14 \\
\hline Diastolic blood pressure $(\mathrm{mmHg})$ & 79 & 12 \\
\hline Habitual Bristol stool scale score & 3 & 1 \\
\hline
\end{tabular}

experienced any aftertaste, feelings of fullness or difficulty in taking the supplement provided. There were no significant differences between the supplement groups for aftertaste (reported by 14-29\% of the participants) or feelings of fullness (reported by $6-14 \%$ of the participants). There was a significant supplement effect on reported difficulty in taking the product $(P=0.006)$, with $32 \%$ of the participants reporting difficulty in taking Bi-07, compared with 6-17\% reporting difficulty in taking the other supplements, with product insolubility being the predominant reported difficulty (twentytwo/twenty-six entries noted under additional comments).

\section{Gut microbiota}

XOS supplementation had a significant effect on faecal bifidobacterial content ( $P=0.008)$ (Table 2). There was no significant supplement effect on total bacterial counts or the other genus-level probes investigated (Table 2). Bi-07 supplementation had a significant effect on the faecal content of this strain $(P=0.007)$ (Table 2$)$, indicative of volunteer compliance with supplement use. Data indicate an additive effect of the provision of XOS with Bi-07 on the change in faecal bifidobacterial content, as the post hoc test revealed that only the XOS + Bi-07 supplement significantly increased bifidobacterial content compared with the placebo. However, data do not support a specific synergistic effect of XOS in combination with Bi-07, as the levels of faecal $B$. lactis were not significantly higher when XOS + Bi-07 was provided compared with those observed when Bi-07 was given alone. No significant supplement effect was observed on faecal dry weight (Table 2). Significant supplement effects were observed on faecal SCFA concentrations (Table 2). XOS + Bi-07 supplementation had a distinct effect on faecal SCFA concentrations when provided in synbiotic combination than when provided individually (Table 2). When XOS or Bi-07 was provided individually, a reduction in acetic and butyric concentrations was apparent, which did not occur when XOS + Bi- 07 was provided. By contrast, faecal isovaleric acid concentrations were increased during the XOS + Bi-07 supplementation period.

\section{Volunteer bowel habit and mood questionnaires}

A significant increase in the number of bowel movements per $\mathrm{d}$ was observed among the volunteers during the XOS supplementation period than during the MDX supplementation period ( $P=0.005$; Table 3 ). No other significant supplement effects on bowel habits or measures of bowel discomfort were observed. XOS supplementation resulted in significantly higher average reported vitality scores $(P=0.001)$ and happiness scores $(P=0 \cdot 05)$ compared with MDX supplementation (Table 4). The proportion of days during which the volunteers reported having less vitality than usual was lower during the XOS supplementation period ( $P=0 \cdot 002$ ), while the proportion of days during which the volunteers reported feeling less happy than usual was higher during the Bi-07 supplementation period than during the XOS supplementation period $(P=0 \cdot 02)$. No significant supplement effects were observed on the self-reported measures of alertness or stress. 
Table 2. Gut microbiota counts, faecal dry weight and faecal SCFA concentrations of volunteers recruited to a double-blind, placebo-controlled, randomised cross-over study during treatment with a candidate prebiotic (xylo-oligosaccharide, XOS, $8 \mathrm{~g} / \mathrm{d}$ ), probiotic (Bifidobacterium animalis subsp. lactis, Bi-07, $10^{9}$ colony-forming units/d) or synbiotic (XOS + Bi-07)

(Mean values and standard deviations)

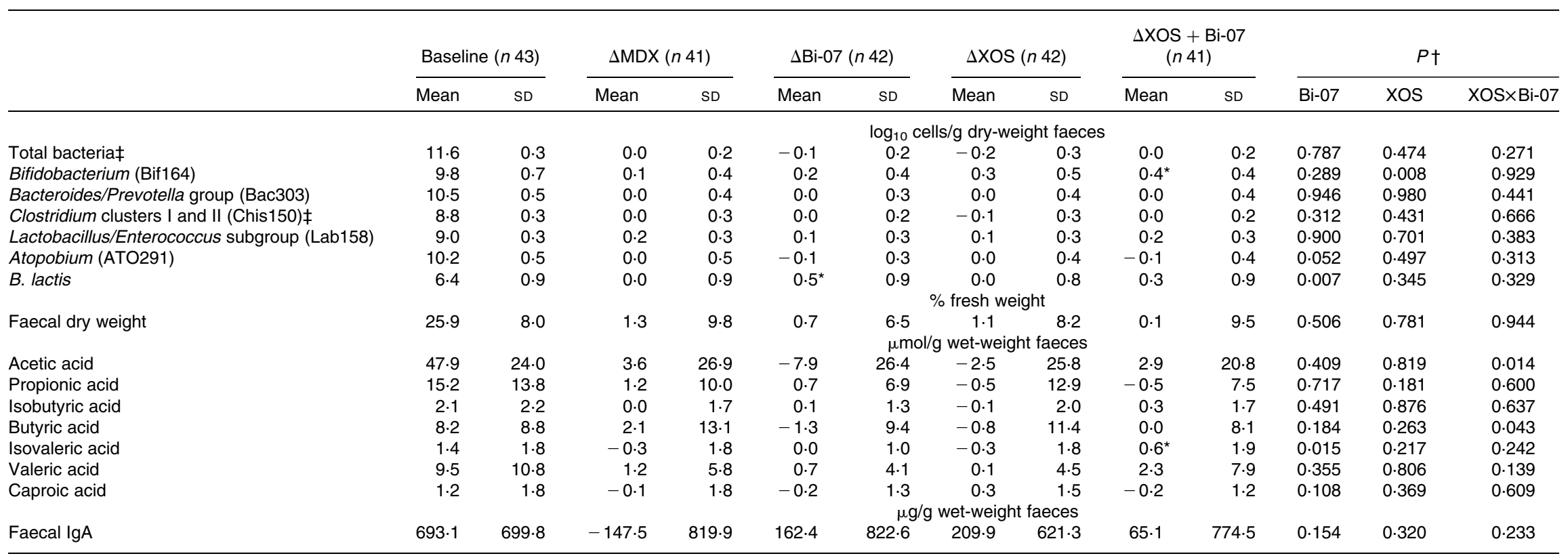

MDX, maltodextrin.

*Mean values were significantly different from those of the MDX group $(P<0.05$; pairwise post hoc comparisons made using contrasts, adjusted using a single-step algorithm)

$\dagger P$ values given are those obtained using linear mixed-effects models $(2 \times 2$ factorial approach) for the presence/absence of the prebiotic (XOS) and probiotic (Bi-07) and their interaction $(X O S \times B i-07)$

$\ddagger n 11$, data from the first treatment period only. 
Table 3. Self-reported bowel habit questionnaire data of volunteers recruited to a double-blind, placebo-controlled, randomised cross-over study during supplementation with a candidate prebiotic (xylo-oligosaccharide, XOS, $8 \mathrm{~g} / \mathrm{d}$ ), probiotic (Bifidobacterium animalis subsp. lactis, Bi-07, $10^{9}$ colony-forming units/d) or synbiotic (XOS $+\mathrm{Bi}-07$ )

(Mean values and standard deviations during the $21 \mathrm{~d}$ supplementation period)

\begin{tabular}{|c|c|c|c|c|c|c|c|c|c|}
\hline & \multicolumn{2}{|c|}{$\operatorname{MDX}(n 39)$} & \multicolumn{2}{|c|}{$\operatorname{XOS}(n 41)$} & \multicolumn{2}{|c|}{$\mathrm{Bi}-07$ ( $n$ 39) } & \multicolumn{2}{|c|}{$\begin{array}{c}\mathrm{XOS}+\mathrm{Bi}-07 \\
(n 39)\end{array}$} & \multirow[b]{2}{*}{$P \dagger$} \\
\hline & Mean & SD & Mean & SD & Mean & SD & Mean & SD & \\
\hline Bowel movements/d & 1.4 & 0.6 & $1.5^{\star}$ & 0.6 & 1.4 & 0.5 & 1.5 & 0.7 & 0.009 \\
\hline \multirow[t]{2}{*}{ Bristol stool scale score } & 3.5 & 0.9 & $3 \cdot 7$ & $0 \cdot 8$ & 3.7 & 1.0 & 3.8 & 0.9 & 0.097 \\
\hline & \multicolumn{9}{|c|}{ Proportion of days reported } \\
\hline$<1$ bowel movement/d & $9 \cdot 4$ & $16 \cdot 1$ & $7 \cdot 8$ & $13 \cdot 0$ & $7 \cdot 7$ & 11.5 & $7 \cdot 1$ & $11 \cdot 3$ & 0.632 \\
\hline$>3$ bowel movements $/ \mathrm{d}$ & $8 \cdot 2$ & $15 \cdot 4$ & $11 \cdot 0$ & $19 \cdot 4$ & $10 \cdot 3$ & $17 \cdot 7$ & $12 \cdot 2$ & $21 \cdot 9$ & 0.554 \\
\hline Bloating & $22 \cdot 4$ & $31 \cdot 3$ & $27 \cdot 6$ & $33 \cdot 1$ & $21 \cdot 8$ & $32 \cdot 6$ & $20 \cdot 9$ & 29.5 & 0.183 \\
\hline Abdominal pain & $15 \cdot 3$ & $22 \cdot 2$ & 16.5 & $28 \cdot 8$ & $17 \cdot 8$ & $26 \cdot 6$ & $13 \cdot 8$ & $23 \cdot 8$ & 0.462 \\
\hline Increased flatulence & $15 \cdot 6$ & $28 \cdot 0$ & $11 \cdot 1$ & $22 \cdot 5$ & $16 \cdot 6$ & $28 \cdot 3$ & 11.4 & $22 \cdot 0$ & 0.388 \\
\hline
\end{tabular}

MDX, maltodextrin.

${ }^{*}$ Mean values were significantly different from those of the MDX group $(P=0.005)$.

$\dagger P$ values reported are those obtained using the Friedman test with Bonferroni corrected post hoc test.

The self-reported incidence of medication use was significantly influenced by supplements (Table 5), but not the duration of medication use (data not presented). The use of analgesics during the $\mathrm{XOS}+\mathrm{Bi}-07$ supplementation period was significantly lower than during the MDX supplementation period $(P=0.012)$, with a similar trend being observed during the XOS supplementation period $(P=0 \cdot 06)$. Further studies are required to identify the change in the incidence or severity of symptoms that underpins this reduced use of analgesic medication, as the present data do not suggest any changes to the incidence or duration of headache, gastrointestinal discomfort/abdominal pain or cold/flu-like symptoms. The self-reported incidence of adverse events or the duration of symptoms reported (data not presented) was not significantly influenced by the supplements (Table 4), though it should be noted that one volunteer withdrew from the study following an adverse reaction to the probiotic supplement.

\section{Plasma lipids}

Fasting HDL concentrations were significantly higher among those volunteers who received the XOS supplement $(P=0.005$; Fig. 1$)$, with an associated trend for a lower total cholesterol:HDL-cholesterol ratio $(P=0 \cdot 06)$. No significant changes in the concentrations of any other plasma lipids were observed (Supplementary Table SA, available online).

Table 4. Self-reported mood questionnaire data of volunteers recruited to a double-blind, placebo-controlled, randomised cross-over study during supplementation with a candidate prebiotic (xylo-oligosaccharide, XOS, $8 \mathrm{~g} / \mathrm{d}$ ), probiotic (Bifidobacterium animalis subsp. lactis, Bi- $07,10^{9}$ colony-forming units $/ \mathrm{d}$ ) or synbiotic (XOS + Bi-07)

(Mean values and standard deviations during the $21 \mathrm{~d}$ supplementation period)

\begin{tabular}{|c|c|c|c|c|c|c|c|c|c|}
\hline & \multicolumn{2}{|c|}{ MDX ( $n$ 39) } & \multicolumn{2}{|c|}{ XOS (n 41) } & \multicolumn{2}{|c|}{ Bi-07 ( $n$ 39) } & \multicolumn{2}{|c|}{$\begin{array}{c}\mathrm{XOS}+\mathrm{Bi}-07 \\
(n 40)\end{array}$} & \multirow[b]{2}{*}{$P \ddagger$} \\
\hline & Mean & SD & Mean & SD & Mean & SD & Mean & SD & \\
\hline \multicolumn{10}{|c|}{ Self-reported score } \\
\hline Vitality & -0.13 & 0.28 & $0.06^{*}$ & 0.24 & -0.02 & 0.28 & -0.01 & 0.25 & 0.003 \\
\hline Happiness & -0.03 & 0.26 & $0.09^{*}$ & 0.22 & -0.03 & 0.38 & 0.02 & 0.18 & 0.034 \\
\hline Alertness & -0.11 & 0.38 & 0.01 & $0 \cdot 13$ & -0.03 & 0.30 & -0.01 & 0.13 & 0.131 \\
\hline Stress & 0.03 & 0.29 & -0.01 & 0.34 & 0.03 & 0.22 & 0.03 & 0.30 & 0.319 \\
\hline \multicolumn{10}{|c|}{ Proportion of days volunteers reported increased } \\
\hline Vitality & $4 \cdot 2$ & $8 \cdot 7$ & 11.9 & $21 \cdot 8$ & 11.4 & 18.5 & $10 \cdot 1$ & $20 \cdot 8$ & 0.353 \\
\hline Happiness & $7 \cdot 7$ & $15 \cdot 9$ & $12 \cdot 7$ & $21 \cdot 2$ & $11 \cdot 8$ & 20.5 & 8.9 & $16 \cdot 1$ & 0.213 \\
\hline Alertness & $3 \cdot 3$ & 8.4 & 4.8 & $10 \cdot 5$ & $8 \cdot 4$ & $19 \cdot 2$ & 5.4 & $12 \cdot 2$ & 0.294 \\
\hline Stress & $11 \cdot 2$ & $16 \cdot 5$ & 9.7 & $13 \cdot 6$ & $9 \cdot 8$ & $16 \cdot 9$ & $10 \cdot 6$ & $19 \cdot 0$ & 0.678 \\
\hline \multicolumn{10}{|c|}{ Proportion of days volunteers reported decreased } \\
\hline Vitality & $15 \cdot 2$ & $21 \cdot 1$ & $6 \cdot 1^{*}$ & $10 \cdot 6$ & $12 \cdot 5$ & $16 \cdot 2$ & $9 \cdot 6$ & 11.8 & 0.007 \\
\hline Happiness & 9.6 & 14.1 & $4 \cdot 1$ & $7 \cdot 9$ & $13.7 \dagger$ & $22 \cdot 8$ & $7 \cdot 3$ & $10 \cdot 2$ & 0.035 \\
\hline Alertness & $11 \cdot 2$ & $20 \cdot 4$ & $4 \cdot 1$ & $9 \cdot 3$ & 11.4 & $20 \cdot 4$ & $6 \cdot 2$ & $9 \cdot 4$ & 0.252 \\
\hline Stress & 8.9 & 21.9 & $7 \cdot 6$ & $18 \cdot 2$ & $7 \cdot 2$ & $12 \cdot 2$ & 8.7 & 20.5 & 0.169 \\
\hline
\end{tabular}

MDX, maltodextrin.

${ }^{*}$ Mean values were significantly different from those of the MDX group $(P<0.05$; Bonferroni corrected post hoc test $)$

† Mean values were significantly different from those of the XOS group $(P=0.02$; Bonferroni corrected post hoc test).

$\ddagger P$ values reported are those obtained using the Friedman test with Bonferroni corrected post hoc test. 
Table 5. Incidence of self-reported medication use and adverse events among volunteers recruited to a double-blind, placebocontrolled, randomised cross-over study during supplementation with a candidate prebiotic (xylo-oligosaccharide, XOS, $8 \mathrm{~g} / \mathrm{d}$ ), probiotic (Bifidobacterium animalis subsp. lactis, Bi-07, $10^{9}$ colony-forming units/d) or synbiotic (XOS + Bi-07) $\dagger$

\begin{tabular}{|c|c|c|c|c|c|}
\hline & $\operatorname{MDX}(n 39)$ & XOS (n 41) & $\mathrm{Bi}-07$ ( $n$ 39) & $\mathrm{XOS}+\mathrm{Bi}-07$ (n 40) & $P \ddagger$ \\
\hline \multicolumn{6}{|l|}{ Medication use } \\
\hline Cold and flu remedies & 9 & 10 & 5 & 4 & 0.415 \\
\hline Analgesics & 48 & 26 & 29 & $16^{*}$ & 0.004 \\
\hline Gastrointestinal medication & 3 & 3 & 0 & 0 & 0.145 \\
\hline Antibiotics & 0 & 1 & 0 & 1 & 0.572 \\
\hline Other (unknown, steroids and sleeping aids) & 0 & 1 & 2 & 1 & 0.801 \\
\hline Antihistamines & 1 & 3 & 2 & 1 & 0.629 \\
\hline No reported medication use & 20 & 24 & 25 & 27 & 0.290 \\
\hline \multicolumn{6}{|l|}{ Adverse events } \\
\hline Cold or flu & 8 & 6 & 10 & 10 & 0.769 \\
\hline Headache & 5 & 19 & 16 & 12 & 0.365 \\
\hline Gastrointestinal symptoms & 4 & 5 & 3 & 3 & 0.849 \\
\hline $\begin{array}{l}\text { Other (inflamed salivary gland, chest pain, } \\
\text { cold sores, sleeping problems, joint pain, } \\
\text { fatigue, night sweats, skin problems and } \\
\text { toothache) }\end{array}$ & 4 & 2 & 4 & 6 & 0.392 \\
\hline No adverse events reported & 27 & 26 & 25 & 28 & 0.673 \\
\hline
\end{tabular}

MDX, maltodextrin.

*Mean values were significantly different from those of the MDX group $(P=0.012)$.

$\dagger$ Data are total number of reported events during each $21 \mathrm{~d}$ supplementation period for each supplement group.

$\ddagger P$ values reported are those obtained using the Friedman test with Bonferroni corrected post hoc test.

\section{Immune parameters}

There were no significant supplement effects on the measures of phagocytosis or oxidative burst or total numbers of leucocytes (data not presented). XOS supplementation resulted in a significantly lower expression of the cell-surface markers CD16/56 on NKT cells ( $P=0 \cdot 027$; Fig. $2(\mathrm{a})$ ). XOS + Bi-07 supplementation resulted in a lower expression of CD19 on $\mathrm{B}$ cells, which may be indicative of changes in B-cell subsets (XOS $\times$ Bi-07, $P=0.009$; Fig. 2(b)). No other significant changes in peripheral blood mononuclear cell phenotypes were observed (Supplementary Table SB, available online), though there was a strong trend for the effect of a XOS $\times \mathrm{Bi}-07$ interaction on the expression of $\mathrm{CD} 3$ on $\mathrm{T}$ cells $(P=0.050)$. A lower expression of $\mathrm{CD} 3$ reduces the potential for $\mathrm{T}$-cell activation, but it is also a normal response to antigen-induced T-cell activation. There were no significant supplement effects on the expression of the activation marker CD69 on T cells after culture with the mitogen ConA (data not presented).

Bi-07 supplementation significantly lowered salivary IgA content $(P=0.04$; Fig. 3). Faecal IgA content was not significantly altered by supplement use (Table 2).

There were significant supplement effects on cytokine secretion in whole-blood samples cultured ex vivo with lipopolysaccharide. Lower IL-4 production ( $P=0.035$; Fig. 4(a)) and higher IL-6 production $(P=0.009$; Fig. $4(\mathrm{~b}))$ were observed during the Bi-07 supplementation period, while lower IL-10 production was observed during the XOS supplementation period $(P=0.049$; Fig. 4(c)). No significant supplement effects were observed on the other cytokines assessed (Supplementary Table SC, available online).

\section{Discussion}

The present placebo-controlled cross-over study investigated the acceptability and efficacy of XOS supplementation in a
European population. XOS provided at $8 \mathrm{~g} / \mathrm{d}$ for a 3 -week period was well tolerated by healthy adults, with no significant effects being observed on the reported symptoms of abdominal pain, bloating or flatulence. XOS supplementation significantly increased self-reported vitality and happiness scores, and the synbiotic combination of $\mathrm{XOS}+\mathrm{Bi}-07$ resulted in significantly lower use of analgesics during the

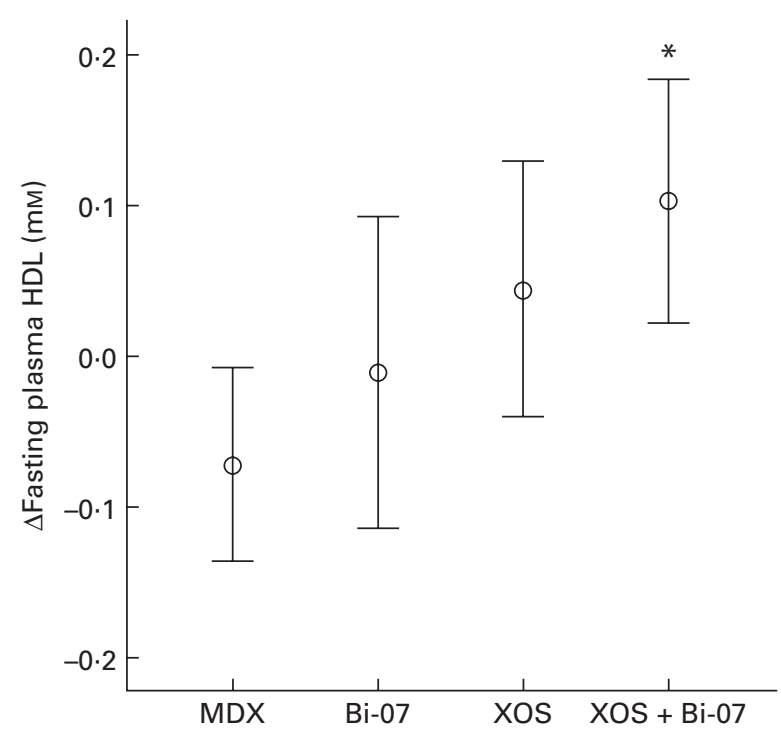

Fig. 1. Significant changes in fasting plasma HDL concentrations among volunteers recruited to a double-blind, placebo-controlled, randomised cross-over study of a candidate prebiotic (xylo-oligosaccharide, XOS, $8 \mathrm{~g} / \mathrm{d}$ ), probiotic (Bifidobacterium animalis subsp. lactis, Bi-07, $10^{9}$ colony-forming units/d) or synbiotic (XOS + Bi-07). Data indicate change from baseline, with mean and $95 \% \mathrm{Cl}$ ( $n$ 11, data from the first treatment period only) being represented by vertical bars. Mean baseline 1.4 (SD 0.5) mM. * Mean values were significantly different from those of the maltodextrin (MDX) group $(P<0.05$; pairwise post hoc comparisons made using contrasts, adjusted using a single-step algorithm). 
(a)

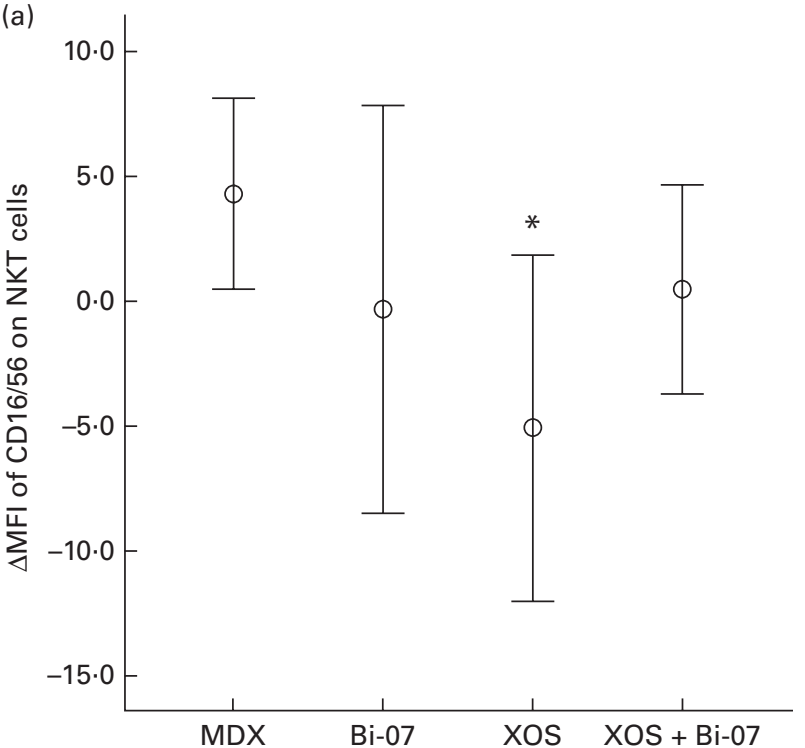

(b)

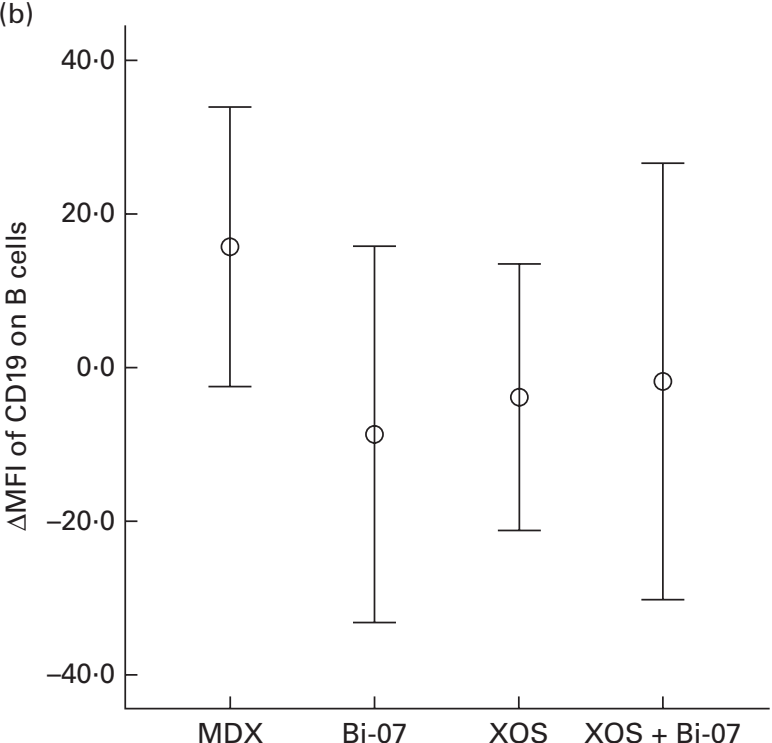

Fig. 2. Significant changes in peripheral blood mononuclear cell phenotypes among volunteers recruited to a double-blind, placebo-controlled, randomised cross-over study of a candidate prebiotic (xylo-oligosaccharide, $\mathrm{XOS}, 8 \mathrm{~g} / \mathrm{d}$ ), probiotic (Bifidobacterium animalis subsp. lactis, Bi-07, $10^{9}$ colony-forming units $/ \mathrm{d}$ ) or synbiotic (XOS + Bi-07). Data indicate change from baseline, with mean and $95 \% \mathrm{Cl}(n 11$, data from the first treatment period only) being represented by vertica bars. (a) Geometric mean fluorescence intensity (MFI) of CD16/56 on natural killer T cells; mean baseline 32.2 (sD 8.9) MFI. (b) Geometric MFI of CD19 on B cells; mean baseline 180 (SD 36). * Mean values were significantly different from those of the maltodextrin (MDX) group ( $P<0.05$; pairwise post hoc comparisons made using contrasts, adjusted using a single-step algorithm).

supplementation period. It may be advantageous to provide XOS as a synbiotic with Bi-07 to simultaneously derive the benefits observed with $\mathrm{Bi}-07$ supplementation while optimising product acceptability, as solubility, in particular, was poor when Bi-07 was provided alone. Therefore, this synbiotic preparation is recommended due to additive effects rather than due to specific synergistic effects.

XOS supplementation resulted in modest, but significant, increases in faecal bifidobacterial counts when provided as either a prebiotic or a synbiotic, confirming the observations reported in studies carried out in Japanese volunteers ${ }^{(16,17)}$. An increased faecal bifidobacterial content may improve colonic health, by competing with potential pathogenic organisms and/or interacting with gut-associated lymphoid tissue ${ }^{(23)}$. Data do not support an additive or synergistic effect of $\mathrm{XOS}+\mathrm{Bi}-07$ supplementation on the gut microbiota, as the increase in faecal B. lactis content observed with Bi-07 treatment was not further enhanced by the co-administration of XOS and nor were the changes observed in bifidobacterial content after XOS + Bi-07 supplementation significantly different from those observed with XOS supplementation alone. The present study utilised fluorescence in situ hybridisation to characterise genus-level changes in the gut microbiota. In future studies, methods such as metagenomics could be utilised to more fully characterise changes in the microbiome and may reveal further changes associated with these dietary supplements. For example, the effects observed on faecal SCFA concentrations during the XOS + Bi-07 supplementation period may reflect a shift from carbohydrate fermentation to protein catabolism in the microbiota, which may be more fully characterised and explored using alternative techniques.
Volunteers who received the XOS supplement exhibited a significant increase in fasting plasma HDL concentrations, with an associated trend for a lower total cholesterol:HDLcholesterol ratio. Lower plasma HDL concentrations have been identified as a significant risk factor for coronary disease $^{(42)}$. The average $0.07 \mathrm{~mm}$ increase in HDL concentrations observed in volunteers receiving the XOS supplement is

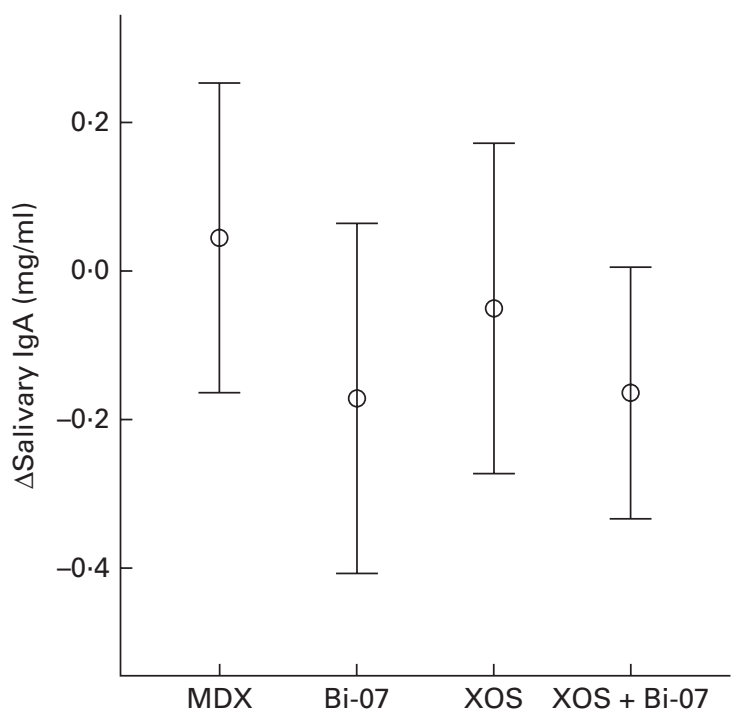

Fig. 3. Significant changes in the salivary $\lg A$ content of volunteers recruited to a double-blind, placebo-controlled, randomised cross-over study during supplementation with a candidate prebiotic (xylo-oligosaccharide, XOS, $8 \mathrm{~g} / \mathrm{d}$ ), probiotic (Bifidobacterium animalis subsp. lactis, Bi-07, $10^{9}$ colonyforming units/d) or synbiotic (XOS + Bi-07). Data indicate change from baseline, with mean and $95 \% \mathrm{Cl}(n 41-42)$ being represented by vertical bars. Mean baseline 1.4 (SD 0.8$) \mathrm{mg} / \mathrm{ml}$. 

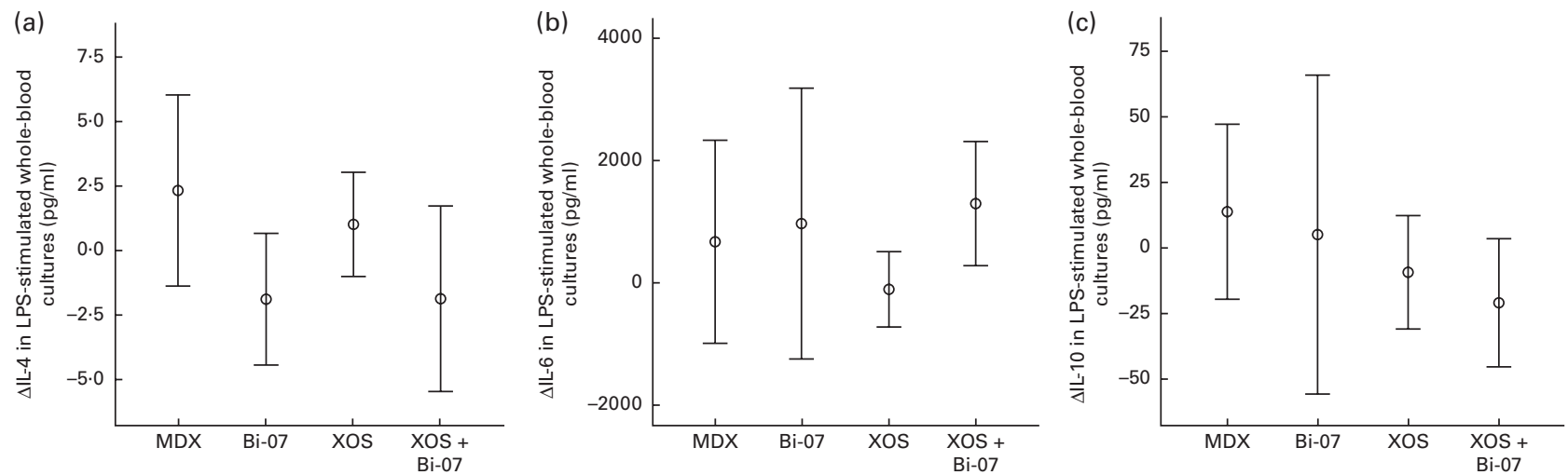

Fig. 4. Significant changes in lipopolysaccharide (LPS)-stimulated cytokine production in whole-blood cultures among volunteers recruited to a double-blind, placebo-controlled, randomised cross-over study of a candidate prebiotic (xylo-oligosaccharide, $\mathrm{XOS}, 8 \mathrm{~g} / \mathrm{d}$ ), probiotic (Bifidobacterium animalis subsp. lactis, $\mathrm{Bi}-07,10^{9}$ colony-forming units/d) or synbiotic (XOS + Bi-07). Data indicate change from baseline, with mean and $95 \% \mathrm{Cl}$ being represented by vertical bars. (a) IL-4, mean baseline 3.7 (SD 7.9) pg/ml ( $n$ 41-42). (b) IL-6, mean baseline 3.5 (SD 3.2) $\mathrm{ng} / \mathrm{ml}(n$ 11, data from the first treatment period only). (c): IL-10, mean baseline 79.7 (SD 88.8$) \mathrm{pg} / \mathrm{ml}(n 11$, data from the first treatment period only).

modest, but a $0 \cdot 1 \mathrm{~mm}$ increase has been estimated to induce a $10 \%$ reduction in CHD risk $^{(43)}$. The proposed mechanisms for the influence of dietary probiotics and prebiotics on plasma lipid concentrations have been generated based on data obtained from in vitro studies and animal models. These include the effect of probiotic bacteria on bile acids, the ability of probiotics to bind to cholesterol, the influence of circulating SCFA on hepatic cholesterol synthesis, the role of prebiotics in the reduction of cholesterol absorption, and the effects of fermentable carbohydrates on gastric emptying rates ${ }^{(44,45)}$. In the present study, no correlations were observed between changes in faecal SCFA concentrations and those to plasma HDL concentrations (data not presented). Further studies are required to identify the mechanisms by which XOS exert this HDL-lowering effect and to determine whether changes in vascular function can be detected. However, the selection of an appropriate 'placebo control' is challenging for a study investigating probiotics and prebiotics. It is possible that the MDX control itself exerted effects on the parameters measured, and data indicate that changes occurring in the placebo group may be driving some of the treatment effects observed on HDL-cholesterol concentrations. This suggests that even a modest increase in daily sugar intake is sufficient to alter HDL-cholesterol concentrations. In addition, only data from the first treatment period were included in the analysis due to carry-over effects observed on this variable. The use of a completely counterbalanced study design, rather than an incompletely counterbalanced measure design, may have mitigated the risk of carry-over and increased the resulting power of this observation.

The effects of both XOS and Bi-07 on the measures of immune status and function were observed. The effects that both probiotics and prebiotics can exert on immune function have been well described in a range of studies including in vitro assessment studies, animal models and human trials ${ }^{(26,46)}$. Probiotics can exert indirect effects on immunity, via mechanisms including alteration of the composition of the gut microbiota, competitive inhibition of potential pathogen-binding sites and improvement of the gut barrier function ${ }^{(46)}$. Probiotics also directly influence signalling pathways in intestinal epithelial cells and dendritic cells, with the potential to induce downstream effects on immune function $^{(47)}$. Prebiotics may influence immune function indirectly, by altering the composition of the gut microbiota or via their own direct effects, such as changes in pathogenassociated molecular patterns presented to the gut-associated lymphoid tissue ${ }^{(23)}$. The present study does not enable conclusions to be drawn about the mechanism of action of XOS and/or Bi-07, but instead provides information on the systemic and mucosal immune measures that have the potential to be modified by supplementation. XOS supplementation induced changes in the cell-surface markers on NKT cells and lowered IL-10 secretion. However, it cannot be excluded that the effects observed on NKT cells were in part influenced by the apparent increase in cell-surface marker expression during the MDX supplementation period. Bi-07 supplementation had significant effects on the systemic markers of immune function, leading to lower IL-4 secretion and salivary IgA content and higher IL- 6 secretion. A XOS $\times$ Bi-07 interaction altered B-cell-surface marker expression. IL-4 secretion is associated with Th2 inflammatory conditions such as asthma and hay fever and promotes B-cell differentiation ${ }^{(47)}$. NKT cells are an important link between innate immunity and adaptive immunity. IL-6 and IL-10 are cytokines with proand anti-inflammatory actions, respectively, and the relative balance of these cytokines is important to prevent excessive inflammation. Salivary IgA is a marker of mucosal immunity, with its secretion being lowered during psychological and physical stress and lower levels being associated with an increased risk of urinary tract infections ${ }^{(48)}$. Taken together, these effects suggest that XOS and Bi-07 have immunostimulatory effects, promoting Th1 responses and lowering Th2 activity. Therefore, these effects may be of benefit to individuals with suppressed Th1 activity, e.g. the elderly, or those with excessive Th2 activity, such as that occurring in atopic disease. The functional consequences of these changes in the measures of immune function should be investigated in suitably designed human studies, preferably using in vivo 
markers of immune function, such as the incidence of allergic rhinitis symptoms or influenza vaccination responses.

The synbiotic $\mathrm{XOS}+\mathrm{Bi}-07$ is a suitable nutritional supplement for healthy adults, leading to increased reported vitality and happiness and reduced use of analgesics, observed among the participants of the present study. Data indicate the potential benefits of both XOS and $\mathrm{Bi}-07$, as a result of their bifidogenic properties, fasting HDL-elevating property and/or immunomodulatory activity. However, data do not support a specific synergistic effect of XOS $+\mathrm{Bi}-07$ on the gut microbiota. Further studies are required to confirm whether the effects of $\mathrm{Bi}-07$ observed on the markers of immune function translate into alterations in the functional or clinical measures of immune function such as the incidence of infection or allergic rhinitis or response to vaccination or whether providing a supplement containing XOS alone or in synbiotic combination with $\mathrm{Bi}-07$ can benefit those with gastrointestinal disorders or inflammatory diseases.

\section{Supplementary material}

To view supplementary material for this article, please visit http://dx.doi.org/10.1017/S0007114513004261

\section{Acknowledgements}

The present study was sponsored by Active Nutrition, DuPont Nutrition and Health (formerly Danisco, Finland), which manufactures and markets the tested probiotic. The employees of Active Nutrition, DuPont Nutrition and Health (formerly Danisco, Finland) contributed to the study design (A. C. O.), conduct of the study (S. D. F.), analysis of the samples and data (H. R. and S. D. F.) and preparation of the manuscript (H. R. and S. D. F.).

The authors' contributions are as follows: G. R. G., K. M. T., R. A. R., A. C. O., C. E. C. and P. Y. designed the research; C. E. C., A. A. F., S. D. F., N. H., Y. N. L. and C. J. S. conducted the research; C. E. C., H. R., E. A. and S. D. F. analysed the data; C. E. C., H. R. and S. D. F. wrote the paper; C. E. C. had primary responsibility for the final content. All authors read and approved the final manuscript.

At the time of the present study, H. R., S. D. F. and A. C. O. were employees of Active Nutrition, DuPont Nutrition and Health (formerly Danisco, Finland), which manufactures and markets the tested probiotic and sponsored the study. None of the other authors have a conflict of interest to report.

\section{References}

1. Gibson GR, Scott KP, Rastall RA, et al. (2010) Dietary prebiotics: current status and new definition. Food Sci Technol Bull Funct Foods 7, 1-19.

2. Imaizumi K, Nakatsu Y, Sato M, et al. (1991) Effects of xylooligosaccharides on blood-glucose, serum and liver lipids and cecum short-chain fatty-acids in diabetic rats. Agr Biol Chem Tokyo 55, 199-205.

3. Koga K \& Fujikawa S (1993) Xylooligosaccharides. In Oligosaccharides: Production, Properties and Applications, Japanese Technology Reviews, 1st ed., pp. 130-143 [T Nakakuki, editor]. Philadelphia, PA: Gordon and Breach Science Publishers.

4. Jaskari J, Kontula P, Siitonen A, et al. (1998) Oat beta-glucan and xylan hydrolysates as selective substrates for Bifidobacterium and Lactobacillus strains. Appl Microbiol Biotechnol 49, 175-181.

5. Kontula P, von Wright A \& Mattila-Sandholm T (1998) Oat bran beta-gluco- and xylo-oligosaccharides as fermentative substrates for lactic acid bacteria. Int J Food Microbiol 45, 163-169.

6. Yong X, Hua J, Quiang Y, et al. (2001) The ability of XOS to promote the proliferation of Bifidobacterium adolescentis Food Sci 22, 15-17.

7. Crittenden R, Karppinen S, Ojanen S, et al. (2002) In vitro fermentation of cereal dietary fibre carbohydrates by probiotic and intestinal bacteria. J Sci Food Agr 82, 781-789.

8. Palframan RJ, Gibson GR \& Rastall RA (2003) Carbohydrate preferences of Bifidobacterium species isolated from the human gut. Curr Issues Intest Microbiol 4, 71-75.

9. Makelainen H, Saarinen M, Stowell J, et al. (2010) Xylo-oligosaccharides and lactitol promote the growth of Bifidobacterium lactis and Lactobacillus species in pure cultures. Benef Microbes 1, 139-148.

10. Rycroft CE, Jones MR, Gibson GR, et al. (2001) A comparative in vitro evaluation of the fermentation properties of prebiotic oligosaccharides. J Appl Microbiol 91, 878-887.

11. Zampa A, Silvi S, Fabiani R, et al. (2004) Effects of different digestible carbohydrates on bile acid metabolism and SCFA production by human gut micro-flora grown in an in vitro semi-continuous culture. Anaerobe 10, 19-26.

12. Makelainen H, Forssten S, Saarinen M, et al. (2010) Xylooligosaccharides enhance the growth of bifidobacteria and Bifidobacterium lactis in a simulated colon model. Benef Microbes 1, 81-91.

13. Campbell JM, Fahey GC Jr \& Wolf BW (1997) Selected indigestible oligosaccharides affect large bowel mass, cecal and fecal short-chain fatty acids, $\mathrm{pH}$ and microflora in rats. $J$ Nutr 127, 130-136

14. Hsu CK, Liao JW, Chung YC, et al. (2004) Xylooligosaccharides and fructooligosaccharides affect the intestinal microbiota and precancerous colonic lesion development in rats. $J$ Nutr 134, 1523-1528

15. Santos A, San Mauro M \& Diaz DM (2006) Prebiotics and their long-term influence on the microbial populations of the mouse bowel. Food Microbiol 23, 498-503.

16. Nakakuki T (2003) Development of functional oligosaccharides in Japan. Trends Glycosci Glyctechnol 15, 57-64.

17. Okazaki M, Fujikawa S \& Matsumoto M (1990) Effect of xylooligosaccharide on the growth of bifidobacteria. Bifidobact Microflora 9, 77-86.

18. Lecerf JM, Depeint F, Clerc E, et al. (2012) Xylo-oligosaccharide (XOS) in combination with inulin modulates both the intestinal environment and immune status in healthy subjects, while XOS alone only shows prebiotic properties. Br J Nutr $\mathbf{1 0 8}$, $1847-1858$.

19. Iino T, Nishijima Y, Sawada S, et al. (1997) Improvement of constipation by a small amount of XOS ingestion in adult women. J Jap Assoc Dietary Fiber Res 1, 19-24.

20. Tateyama I, Hashii K, Johno I, et al. (2005) Effect of xylooligosaccharide intake on severe constipation in pregnant women. J Nutr Sci Vitaminol (Tokyo) 51, 445-448.

21. Gibson GR, Probert HM, Loo JV, et al. (2004) Dietary modulation of the human colonic microbiota: updating the concept of prebiotics. Nutr Res Rev 17, 259-275.

22. Roberfroid M (2007) Prebiotics: the concept revisited. J Nutr 137, 830S-837S. 
23. Roberfroid M, Gibson GR, Hoyles L, et al. (2010) Prebiotic effects: metabolic and health benefits. BrJ Nutr 104, S1-S63.

24. Ebersbach T, Jorgensen JB, Heegaard PM, et al. (2010) Certain dietary carbohydrates promote Listeria infection in a guinea pig model, while others prevent it. Int $J$ Food Microbiol 140, 218-224.

25. Ebersbach T, Andersen JB, Bergstrom A, et al. (2012) Xylooligosaccharides inhibit pathogen adhesion to enterocytes in vitro. Res Microbiol 163, 22-27.

26. Lomax AR \& Calder PC (2009) Probiotics, immune function, infection and inflammation: a review of the evidence from studies conducted in humans. Curr Pharm Des 15, 1428-1518.

27. Ringel-Kulka T, Palsson OS, Maier D, et al. (2011) Probiotic bacteria Lactobacillus acidophilus NCFM and Bifidobacterium lactis $\mathrm{Bi}-07$ versus placebo for the symptoms of bloating in patients with functional bowel disorders: a double-blind study. J Clin Gastroenterol 45, 518-525.

28. Leyer GJ, Li S, Mubasher ME, et al. (2009) Probiotic effects on cold and influenza-like symptom incidence and duration in children. Pediatrics 124, e172-e179.

29. Rastall RA \& Maitin V (2002) Prebiotics and synbiotics: towards the next generation. Curr Opin Biotechnol 13, 490-496.

30. Erdfelder E, Faul F \& Buchner A (1996) GPOWER: a general power analysis program. Behav Res Methods Instrum Comput 28, $1-11$.

31. Gill HS, Rutherfurd KJ, Cross ML, et al. (2001) Enhancement of immunity in the elderly by dietary supplementation with the probiotic Bifidobacterium lactis HN019. Am J Clin Nutr 74, 833-839.

32. Roessler A, Friedrich U, Vogelsang H, et al. (2008) The immune system in healthy adults and patients with atopic dermatitis seems to be affected differently by a probiotic intervention. Clin Exp Allergy 38, 93-102.

33. Seidel C, Boehm V, Vogelsang $\mathrm{H}$, et al. (2007) Influence of prebiotics and antioxidants in bread on the immune system, antioxidative status and antioxidative capacity in male smokers and non-smokers. Br J Nutr 97, 349-356.

34. Roller M, Clune Y, Collins K, et al. (2007) Consumption of prebiotic inulin enriched with oligofructose in combination with the probiotics Lactobacillus rhamnosus and Bifidobacterium lactis has minor effects on selected immune parameters in polypectomised and colon cancer patients. Br J Nutr 97, 676-684.
35. Kang M, Ragan BG \& Park JH (2008) Issues in outcomes research: an overview of randomization techniques for clinical trials. J Athl Train 43, 215-221.

36. Costabile A, Kolida S, Klinder A, et al. (2010) A doubleblind, placebo-controlled, cross-over study to establish the bifidogenic effect of a very-long-chain inulin extracted from globe artichoke (Cynara scolymus) in healthy human subjects. Br J Nutr 104, 1007-1017.

37. Ventura M, Reniero R \& Zink R (2001) Specific identification and targeted characterization of Bifidobacterium lactis from different environmental isolates by a combined multiplexPCR approach. Appl Environ Microbiol 67, 2760-2765.

38. Apajalahti JH, Kettunen H, Kettunen A, et al. (2002) Cultureindependent microbial community analysis reveals that inulin in the diet primarily affects previously unknown bacteria in the mouse cecum. Appl Environ Microbiol 68, 4986-4995.

39. Richardson AJ, Calder AG, Stewart CS, et al. (1989) Simultaneous determination of volatile and non-volatile acidic fermentation products of anaerobes by capillary gas-chromatography. Lett Appl Microbiol 9, 5-8.

40. Costabile A, Fava F, Roytio H, et al. (2012) Impact of polydextrose on the faecal microbiota: a double-blind, crossover, placebo-controlled feeding study in healthy human subjects. Br J Nutr 108, 471-481.

41. Hothorn T, Bretz F \& Westfall P (2008) Simultaneous inference in general parametric models. Biom J 50, 346-363.

42. Shepherd J (2005) Raising HDL-cholesterol and lowering CHD risk: does intervention work? Eur Heart J 7, F15-F22.

43. Gordon DJ, Probstfield JL, Garrison RJ, et al. (1989) Highdensity lipoprotein cholesterol and cardiovascular disease. Four prospective American studies. Circulation 79, 8-15

44. Delzenne NM \& Williams CM (2002) Prebiotics and lipid metabolism. Curr Opin Lipidol 13, 61-67.

45. Ooi L-G \& Liong M-T (2010) Cholesterol-lowering effects of probiotics and prebiotics: a review of in vivo and in vitro findings. In J Mol Sci 11, 2499-2522.

46. Lomax AR \& Calder PC (2009) Prebiotics, Immune function, infection and inflammation: a review of the evidence. $\mathrm{BrJ}$ Nutr 101, 633-658.

47. Bermudez-Brito M, Plaza-Díaz J, Muñoz Quezada S, et al. (2012) Probiotic mechanisms of action. Ann Nutr Metab 61, 160-174.

48. Albers R, Antoine JM, Bourdet-Sicard R, et al. (2005) Markers to measure immunomodulation in human nutrition intervention studies. Br J Nutr 94, 452-481. 